\title{
Uma revisão sistemática da literatura sobre o Problema de Roteirização de Veículos Capacitados com Restrições de Carregamento Bidimensional (2L-CVRP)
}

\author{
Bianca Passos Arpini ${ }^{1}$ e Rodrigo Alvarenga Rosa ${ }^{2}$
}

\begin{abstract}
Resumo: Roteirização e carregamento são dois aspectos centrais na gestão de distribuição logística. Recentemente, o problema integrado de roteirização e carregamento tem atraído a atenção da comunidade de pesquisa operacional. Esse interesse se deve não só a seu enorme potencial de aplicação em situações reais, mas também aos desafios metodológicos para sua solução, pois se trata da combinação de dois problemas de elevada complexidade computacional. Este artigo tem por objetivo realizar uma revisão sistemática das principais publicações relacionadas ao Problema de Roteirização de Veículos Capacitados com Restrições de Carregamento Bidimensional (Capacitated Vehicle Routing Problem with Two-dimensional Loading Constraints - 2L-CVRP). O intuito é analisar o que foi publicado até o momento e identificar abordagens de solução, características incorporadas ao problema e lacunas de pesquisa que ainda não foram tratadas.

Palavras-chave: problemas de roteirização de veículos com restrição de carregamento bidimensional, problemas 2L-CVRP, problema de roteirização de veículos.
\end{abstract}

\begin{abstract}
Routing and loading are two central aspects in logistics distribution management. Recently, the integrated routing and loading problem has attracted the attention of the Operations Research community. Such an interest is not only due to their enormous application potential in real situations, but also due to the challenges for their solution, because it is the combination of two problems of high computational complexity. This article aims to conduct a systematic review of key publications related to the Capacitated Vehicle Routing Problem with Two-Dimensional Loading Constraints (2L-CVRP). The objective is to analyze what has been published so far and identify approaches to solution features built into the problem and research gaps that have not been treated.
\end{abstract}

Keywords: vehicle routing problem with two-dimensional loading constraints, 2L-CVRP problem, vehicle routing problem.

\section{INTRODUÇÃO}

Nos sistemas logísticos, é essencial planejar e gerenciar as atividades de transportes de cargas, pois os custos relativos ao transporte representam uma grande parcela dos custos logísticos totais (Labadie e Prins, 2012).

Nesse sentido, há dois aspectos centrais na gestão de distribuição logística (Iori e Martello, 2013): problemas de roteirização de veículos, usados para determinar a rota ótima e problemas de carregamento, usados para definir a melhor maneira de carregar mercadorias dentro dos veículos utilizados no transporte. Em geral, a construção de resoluções para essas duas áreas de pesquisa têm sido desenvolvidas separadamente. Contudo, recentemente, abordagens de resolução integrada dos dois problemas têm sido propostas na literatura. Visando a criação de rotas e a arrumação bidimensional de cargas, foi proposto na literatura o Problema de Roteirização de Veículos Capacitados com Restrições de Carregamento Bidimensional (Capacitated Vehicle Routing Problem with Two-dimensional Loading Constraints - 2L-CVRP). O 2L-CVRP é uma combinação de dois problemas NP-hard: o Capacitated Vehicle Routing Problem (CVRP) e o Two-dimensional Bin Packing Problem (2BPP) (Leung et al., 2011).

Este artigo tem por objetivo realizar uma revisão sistemática das principais publicações relacionadas ao $2 \mathrm{~L}$ -

\footnotetext{
1 Bianca Passos Arpini, Mestrado em Engenharia Civil - Transportes,

UFES. (bianca.p.arpini@gmail.com)

2 Rodrigo Alvarenga Rosa, Mestrado em Engenharia Civil - Transportes,

UFES. (rodrigoalvarengarosa@gmail.com)
}

Manuscrito recebido em 21/11/2014 e aprovado para publicação em $24 / 08 / 2016$

Este artigo é parte de TRANSPORTES v. 25, n. 1, 2017. ISSN: 2237-1346 (online). DOI:10.14295/transportes.v25i1.867

TRANSPORTES v. 25, n. 1 (2017), p. 61-72
CVRP. O intuito é analisar o que foi estudado até o momento e identificar abordagens de solução, características do CVRP e do 2BPP incorporadas ao 2L-CVRP e lacunas que ainda não foram preenchidas nessa classe de problema. Existem quatro artigos de revisão da literatura sobre problemas integrados de roteirização e carregamento (Wang et al., 2009; Iori e Martello, 2010; Ranck Júnior e Yanasse, 2010; Iori e Martello, 2013), no entanto, este artigo tem como diferencial, em relação aos quatro artigos citados, os seguintes pontos: a inclusão de nove publicações que até então não haviam sido consideradas nas revisões acerca do $2 \mathrm{~L}-\mathrm{CVRP}$ (Azevedo et al., 2009; Muñoz, 2011; Hamdi-Dhaoui et al., 2012; Bin et al., 2013; Côté et al., 2013; Dominguez et al., 2014a,b; Abdal-Hammed et al., 2014; Wei et al., 2015); o uso de uma metodologia específica para efetuar a revisão; a realização de análises que incluem a distribuição geográfica e anual dos artigos; e a síntese das características e categorias do 2L-CVRP e do ambiente de desenvolvimento das abordagens de solução adotadas.

O restante artigo é organizado como segue: na Seção 2 é dada a definição do 2L-CVRP; na Seção 3 apresenta-se a metodologia utilizada para realizar a revisão sistemática; na Seção 4 têm-se a descrição da revisão sistemática e os resultados obtidos, que incluem uma análise das contribuições existentes até o momento; e a Seção 5 contém as considerações finais.

\section{PROBLEMA DE ROTEIRIZAÇÃO DE VEÍCULOS COM RESTRIÇÕES DE CARREGAMENTO EM DỦAS DIMENSÕES}

O 2L-CVRP pode ser descrito da seguinte maneira (Iori et al., 2007; Gendrau et al., 2008; Zachariadis et al., 2009; Fuellerer et al., 2009; Azevedo et al., 2009; Araujo, 
2010; Leung et al. 2010; Leung et al., 2011; Duhamel et al., 2011; Zachariadis et al., 2013): No 2L-CVRP há um depósito central, um conjunto de clientes, uma frota homogênea de veículos e um conjunto de itens retangulares demandados para entregar aos clientes. A cada veículo estão associados uma capacidade de peso e uma superfície de carregamento de duas dimensões. Cada item possui um tamanho e um peso determinado e não pode ser empilhado. Todos os itens de um mesmo cliente devem ser atribuídos a um único veículo. Todos os veículos, por sua vez, devem começar e terminar suas rotas no depósito central e os itens transportados devem possuir uma arrumação viável dentro da superfície de carregamento desses veículos. O objetivo do problema é colocar e arrumar os itens dentro dos veículos e entregá-los aos clientes, por meio de uma rede de transportes, com mínimo custo total.

Seja um grafo não orientado completo $G=(V, E)$, em que $V$ indica o conjunto de $n+1$ vértices, correspondendo ao depósito central (vértice 0), e ao conjunto de clientes (vértices $=1,2, \ldots, n$ ). Para cada aresta $e \in E$ está associado um custo $c_{e}$. Há um conjunto de $K$ veículos disponíveis no depósito, caracterizados por uma capacidade de peso $D$ e uma superfície retangular de carregamento de largura $W$ e comprimento $L$. Cada veículo é acessível por apenas um único lado (parte traseira) para operações de carga e descarga. A área total da superfície de carregamento é denotada por $A=W L$. A cada cliente $i \quad(i=1,2, \ldots, n)$ está associado um conjunto de $m_{i}$ itens retangulares, cujo peso total é $d_{i}$. Cada item tem largura $w_{i p}$ e comprimento $l_{i p}\left(p=1, \ldots, m_{i}\right)$. A área total dos itens demandados pelo cliente $i$ é denotada por $a_{i}=\sum_{p=1}^{m_{i}} w_{i p} l_{i p}$. Por fim, tem-se que $M=\sum_{i=1}^{n} m_{i}$ é o número total de itens.

O objetivo do 2L-CVRP é definir os clientes que serão atendidos por cada rota tal que o custo de transporte total seja minimizado, e que satisfaça as seguintes restrições:

a. O tamanho do conjunto de rotas geradas não deve exceder o número de veículos disponíveis (no máximo uma rota por veículo);

b. Cada rota começa e termina no depósito central;

c. Todos os clientes devem ser visitados apenas uma única vez;

d. O peso total de todos os itens em cada rota não deve exceder a capacidade do veículo;

e. A demanda de cada cliente deve ser totalmente satisfeita;

f. Todos os itens de um dado cliente devem ser carregados em um mesmo veículo. Em outras palavras, entregas fracionadas não são permitidas;

g. O carregamento deve ser ortogonal, ou seja, os itens devem ser carregados com seus lados paralelos aos lados da superfície de carregamento;

h. Todos os itens de cada cliente devem estar completamente contidos na superfície de carregamento do veículo; i. Não poderá ocorrer sobreposição de itens dentro da superfície de carregamento dos veículos.

Além das restrições mencionadas, há outros dois aspectos a serem considerados: um referente à possibilidade de reacomodar ou não a carga ao longo da rota e outro relativo à orientação do item, gerando novas terminologias para o problema.

No que tange ao primeiro ponto, há duas variantes: o 2L-CVRP irrestrito e o 2L-CVRP sequencial. No 2LCVRP sequencial a disposição da carga no veículo deve obedecer à ordem inversa de descarga, análogo a um sistema LIFO (Last In - First Out) (Gendrau et al., 2008; Zachariadis et al., 2009; Fuellerer et al., 2009; Araujo, 2010; Leung et al., 2011). No 2L-CVRP irrestrito a disposição dos itens não inclui a restrição de sequenciamento e corresponde, por exemplo, à situação em que veículos podem ser descarregados verticalmente com um guindaste (Khebbache-Hadji et al., 2013).

No que concerne à orientação do item, pode haver um carregamento orientado ou não orientado. No orientado os itens possuem uma orientação fixa, não podendo ser rotacionados. No não orientado os itens podem ser rotacionados em $90^{\circ}$ (Fuellerer et al., 2009).

Na literatura, Fuellerer et al. (2009) foram os primeiros a propor uma classificação de quatro categorias relacionada com a configuração do carregamento incluindo a possibilidade de rotacionar o item. A partir disso, são definidas quatro classes de problemas bidimensionais (Fuellerer et al., 2009; Duhamel et al., 2011; Zachariadis et al., 2013):

- 2|SO|L: Two-Dimensional Sequential Oriented Loading ou carregamento orientado sequencial bidimensional - esta classe inclui restrição de sequenciamento e os itens tem orientação fixa;

- 2|SR|L: Two-Dimensional Sequential Rotated Loading ou carregamento rotacionado sequencial bidimensional - esta classe inclui restrição de sequenciamento e os itens podem ser rotacionados;

- 2|UO|L: Two-Dimensional Unrestricted Oriented Loading ou carregamento orientado irrestrito bidimensional - esta classe não inclui restrição de sequenciamento e os itens têm orientação fixa;

- 2|UR|L: Two-Dimensional Unrestricted Rotated Loading ou carregamento rotacionado irrestrito bidimensional - esta classe não inclui restrição de sequenciamento e os itens podem ser rotacionados.

O 2L-CRVP é modelado usando uma adaptação da formulação clássica de dois índices do CVRP de Toth e Vigo (2002). Seja $z_{e}$ uma variável binária para todo $e \in E$, que assume valor 1 , se e somente se, uma veículo viaja ao longo da aresta $e$ em uma rota viável, e zero caso contrário. Dado um subconjunto de clientes $S \subseteq V, d(S)$ é o peso total de todos os clientes em $S$, ou seja, $d(S)=\sum_{i \in S} d_{i}$. Denote por $a(S)$ a área total dos itens dos clientes em $S$. Seja $\delta(S)$ o conjunto de arestas em $G$ com exatamente um vértice em $S$ e seja $\gamma(S)$ o conjunto de arestas em $G$ com am- 
bos os vértices em $S$. Seja $r(S)$ o número mínimo de veículos necessários para suprir a demanda de $S$. Além disso, dada uma rota viável $(S, \sigma)$, denota-se por $E(S, \sigma)$ o conjunto de arestas em cada rota. Mais especificamente $E(S, \sigma)=\{(0, \sigma(1)),(\sigma(1), \sigma(2)), \ldots,(\sigma(|s|), 0)\}$. Por fim, denota-se por $\sum(S)$ a coleção de sequencias $\sigma$ tal que $(S, \sigma)$ é uma rota viável. A formulação da programação inteira é (IORI et al. 2007):

Função Objetivo:

$$
\min \sum_{e \in E} c_{e} z_{e}
$$

\section{Restrições}

$$
\begin{array}{ll}
\sum_{e \in \delta(i)} z_{e}=2 & \forall i \in V \backslash\{0\} \\
\sum_{e \in \delta(0)} z_{e}=2 K & \\
\sum_{e \in \delta(S)} z_{e} \geq 2 r(S) & \forall S \subseteq V \backslash\{0\}, S \neq \varnothing \\
\sum_{e \in E(S, \sigma)} z_{e} \leq|S|-1 & \forall(S, \sigma) \mid \sigma \notin \sum(S) \\
z_{e} \in\{0,1\} & \forall e \in E \backslash \delta(0) \\
z_{e} \in\{0,1,2\} & \forall e \in \delta(0)
\end{array}
$$

A função objetivo expressa em (1) trata da minimização do custo de transporte. As restrições (2) garantem que cada cliente é visitado exatamente uma vez. As restrições (3) asseguram que exatamente ${ }_{K}$ veículos são usados.

As restrições representadas por (4), conhecidas como restrições de capacidade-corte, impõem tanto conectividade quanto viabilidade de solução com relação às condições expressas nos itens (g), (h), (i), ou seja, capacidade, respeito ao limitede espaço de carregamento e sobreposição de itens, respectivamente.

O cálculo de $r(S)$ é $N P$-hard, pois equivale a encontrar a solução do 2BPP, dados os itens dos clientes e uma superfície de carregamento dos veículos. Por isso, é usual na literatura substituir $r(S)$ por um limite inferior sobre o seu valor, o que é obtido por relaxação. Em geral, adota-se $r^{\prime}(S)=\max \left\{\left[\frac{d(S)}{D}\right],\left[\frac{a(S)}{A}\right]\right\}$, conhecidas como Desigualdades de Capacidade Arredondadas (Rounded Capacity Inequalities), em que o primeiro termo é o limite inferior contínuo do 1BPP associado com o peso dos itens dos clientes, e o segundo é o limite inferior contínuo do 2BPP relacionado com a área total ocupada pelos itens dos clientes em $S$.

As restrições (5), conhecidas como restrições de caminho inviável (infeasible-path constraints), consideram os requisitos da sequência de carregamento, eliminando sequências $\sigma$ não viáveis associadas com um conjunto de clientes $S$. Ressalta-se que tal restrição só será válida para problemas do tipo $2|\mathrm{SO}| \mathrm{L}$ e $2|\mathrm{SR}| \mathrm{L}$, que contém a restrição de sequenciamento. Por fim as restrições (6) impõem que as variáveis associadas com as arestas que ligam dois clientes são binárias, e as restrições (7) estabelecem que as variáveis relacionadas com arestas que conectam os clientes ao depósito também podem assumir o valor dois (representando, assim, uma rota de um único cliente).

\section{METODOLOGIA}

De acordo com Okoli e Schabram (2010) uma revisão sistemática da literatura, sistematiza, explicita e executa um método para identificar, avaliar e sumarizar o estado da arte de uma área de conhecimento específica. Brereton et al. (2007) apresentaram um método de revisão sistemática de literatura constituído por três fases: planejamento, realização e documentação (Figura 1).

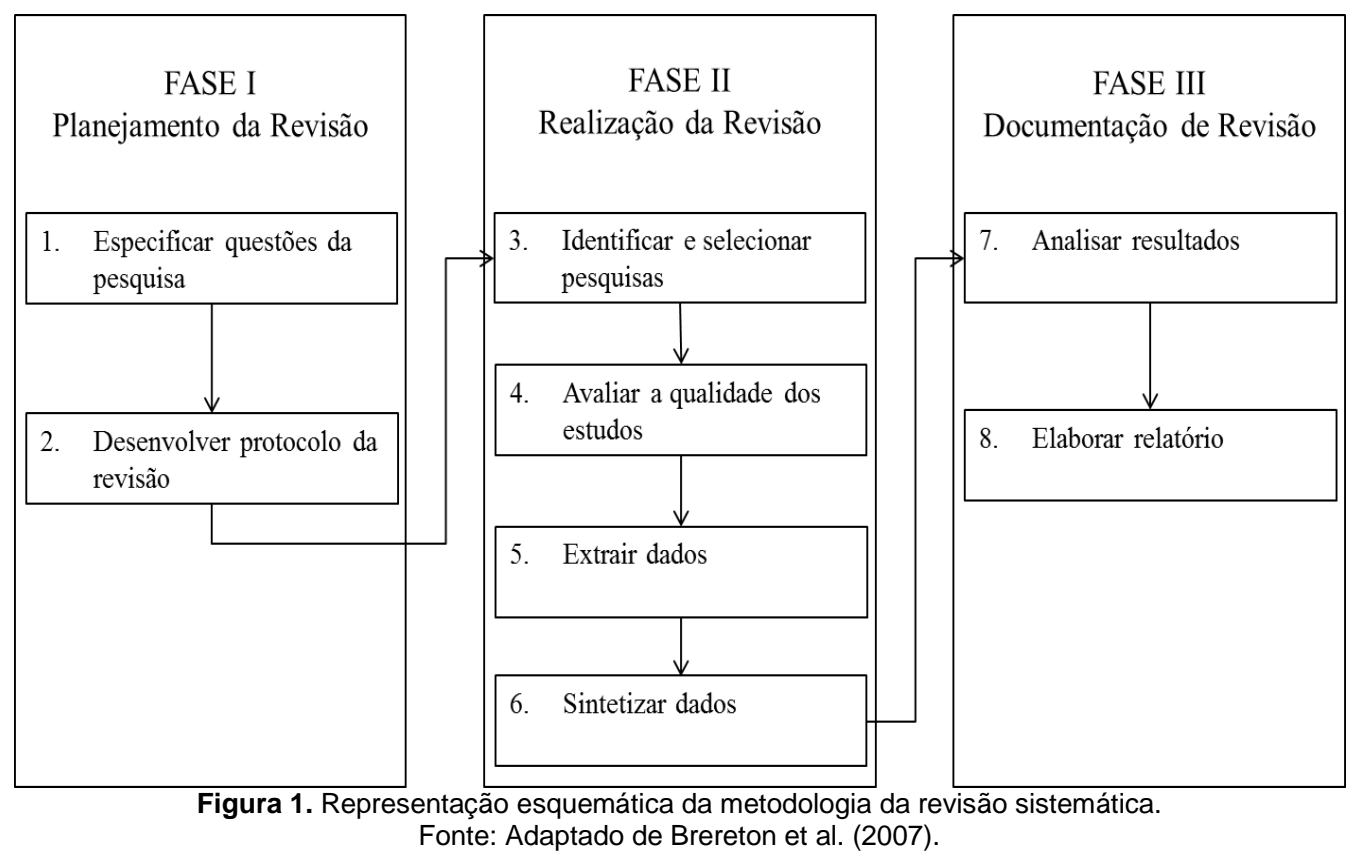

TRANSPORTES, v. 25, n. 1 (2017), p. 61-72 
A Figura 1 mostra que a Fase I é composta por duas etapas: Especificar questões da pesquisa e Desenvolver protocolo da revisão. Nesta fase, define-se o tema abordado, os objetivos e o procedimento a ser adotado para realizar a revisão. A Fase II contém quatro etapas: Identificar e selecionar pesquisas; Avaliar a qualidade dos estudos; Extrair dados; Sintetizar dados. Nesta fase, elaboram-se critérios para selecionar e avaliar os trabalhos identificados e organiza-se a informação extraída. A Fase III apresenta duas etapas: Analisar resultados e Elaborar relatório.

\section{APLICAÇÃO DA METODOLOGIA E RESULTADOS ENCONTRADOS}

Nesta seção apresentam-se a aplicação da metodologia ilustrada na Figura 1 bem como os resultados obtidos.

\subsection{Fase I: Planejamento da Revisão} Fase I.

Esta subseção descreverá as etapas relacionadas à

\subsubsection{Etapa 1: Especificar questões da pesquisa}

Esta revisão visa responder às seguintes questões relativas ao 2L-CVRP: 1) Quais as abordagens de solução empregadas?; 2) Quais características são incorporadas ao problema?; 3) Quais as lacunas existentes na literatura?.

\subsubsection{Etapa 2: Desenvolver protocolo da revisão}

Para identificar as pesquisas efetuaram-se buscas nas bases Science Direct, Wiley Online Library, IEEE Xplore, Scielo, Scopus e Domínio Público, além da plataforma de busca do Google Scholar, utilizando como palavras-chave, combinadas ou não, "Vehicle routing", "loading constraints", "bin packing", "two-dimensional packing", "2LCVRP", "two-dimensional loading capacitated vehicle routing problem", "problema de roteirização de veículos com restrições de carregamento bidimensional", "roteirização", "carregamento bidimensional". As palavras-chave foram procuradas no título, no resumo e nas palavras-chave dos estudos. Considerou-se o período de 2007 a 2015 e não houve limitação quanto ao país de publicação. Para selecionar as pesquisas identificadas, foram considerados artigos científicos, publicados em periódicos ou em congressos; teses; dissertações; livros e relatórios de pesquisa relacionados às áreas de transportes e pesquisa operacional que tratam do problema de roteirização integrado com carregamento. Foram pesquisados artigos nos idiomas inglês, espanhol, português e francês. As monografias de curso de pósgraduação e trabalhos de curso de graduação foram excluídas da pesquisa.

Para avaliar a qualidade dos estudos, fez-se a leitura completa dos trabalhos selecionados e foram utilizados critérios de inclusão específicos. O critério de inclusão englobou estudos que contivessem abordagens de solução para o 2L-CVRP e realização de testes em instâncias de referência da literatura.

A partir dos estudos que atenderam aos critérios expostos anteriormente, extraíram-se dados relativos à fonte de pesquisa (artigos de periódico, artigos de congresso e ou- tros); ao ano da publicação; ao país onde a pesquisa foi desenvolvida; às características de CVRP e 2BPP incorporadas ao problema; e à abordagem de solução empregada.

\subsection{Fase II: Realização da Revisão}

Nesta subseção abordam-se as quatro etapas associadas à Fase II.

\subsubsection{Etapa 3: Identificar e Selecionar Pesquisas}

A identificação e seleção dos trabalhos seguiram os critérios apresentados na seção 4.1.2, resultando, inicialmente, na identificação de 29 estudos.

\subsubsection{Etapa 4: Avaliar a qualidade dos estudos}

A avaliação da qualidade foi feita por meio da leitura completa dos trabalhos selecionados e utilizando-se os critérios de inclusão descritos na seção 4.1.2. Esta etapa levou à exclusão de seis artigos, que não atendem aos critérios de inclusão estabelecidos, sendo considerados 23 trabalhos para a realização da revisão sistemática de literatura. Após isso, extraíram-se os dados.

\subsubsection{Etapa 5: Extrair dados}

Quanto às fontes de pesquisa, 61\% dos artigos científicos foram encontrados em periódicos e $26 \%$ foram publicados em congressos. Há $13 \%$ de publicações, que correspondem a três documentos, provenientes de outras fontes, sendo elas: Lecture Notes in Computer Science (LNCS) (Strodl et al., 2010), Dissertação da Pontifícia Universidade Católica do Chile (PUC Chile) (Muñoz, 2011) e Relatório de Pesquisa do CIRRELT (Côté et al., 2013 ).

Os periódicos com maior concentração de artigos são o European Journal of Operational Research (EJOR) e o Computers \& Operations Research (COR), com quatro e três publicações, respectivamente. No que se refere à quantidade de artigos por congresso, há apenas um artigo para cada um dos seguintes congressos: First CPAIOR Workshop on Bin Packing and Placement Constraints (BPPC'08), Simpósio Brasileiro de Pesquisa Operacional (SBPO 2009), $9^{\circ}$ Conférence Internationale de Modélisation, Optimisation et Simulation (MOSIM'12), International MultiConference of Engineers and Computer Scientists (IMECS 2012), International Conference on Management Science \& Engineering (ICMSE 2013) e International Conference on Control, Decision and Information Technologies (CoDIT 2014).

Em relação ao período abrangido na pesquisa, a média de publicação anual foi de dois artigos até o ano de 2012. Em contrapartida, o ano de 2013 concentrou a maior quantidade de publicações em um ano, totalizando seis artigos. No que tange à distribuição geográfica dos estudos, a maioria das publicações é proveniente da China, totalizando seis artigos.

\subsubsection{Etapa 6: Sintetizar dados}

A síntese dos artigos selecionados, com enfoque na abordagem de solução usada, características do CVRP e 2BPP incorporadas, bem como as categorias do 2L-CVRP tratadas em cada artigo são apresentadas a seguir. 
Ressalta-se que os artigos foram agrupados em duas classes: 2L-CVRP sem variantes e 2L-CVRP com variantes.

O 2L-CVRP foi introduzido por Iori et al. (2007), que apresentaram um modelo de Programação Linear Inteira gerado a partir da adaptação do modelo clássico de CVRP de Toth e Vigo (2002). No que tange ao método de solução para o 2L-CVRP, os autores propuseram uma abordagem exata para o problema, aplicando um algoritmo Branchand-Cut (B\&C) para minimizar o custo de roteamento, e um algoritmo Branch-and-Bound (B\&B) para verificar a viabilidade de carregamento.

Para testar a abordagem exata, Iori et al. (2007) criaram instâncias para o 2L-CVRP a partir das instâncias do CVRP de Toth e Vigo (2002). Essas instâncias incluem características do $2 \mathrm{BPP}$, como a largura e comprimento da superfície de carregamento e dos itens de cada cliente, além dos parâmetros inerentes ao CVRP, tais como capacidade dos veículos; pesos associados com a demanda de cada cliente; número de veículos e custos das arestas, que são calculados pela distância euclidiana entre dois pontos. Considera-se que cada instância tem cinco classes, sendo que cada uma corresponde a uma forma diferente de gerar os itens associados a cada cliente. Cada um dos itens possui uma forma, nomeadas vertical (o intervalo das larguras dos itens é maior que o intervalo dos comprimentos), homogênea (a largura e o comprimento dos itens são sorteados no mesmo intervalo) e horizontal (o intervalo dos comprimentos dos itens é maior que o intervalo de larguras).

A Tabela 1 apresenta informações a respeito das classes das instâncias, em que $m_{i}$ representa os itens pertencentes ao cliente $i$ e $l_{i p}$ e $w_{i p}$ se referem ao comprimento e à largura de cada item, respectivamente. Para todas as instâncias, a superfície de carregamento dos veículos tem $L=40$ e $W=20$.

A primeira classe é equivalente à instância original do CVRP, na qual cada cliente tem apenas um item e todos itens têm ambas as dimensões iguais a 1. Para cada classe $r=2, \ldots, 5$ o número de itens gerados para cada cliente é um valor inteiro uniformemente aleatório no intervalo $[1, r]$.
O algoritmo global de Iori et al. (2007) é capaz de resolver à otimalidade instâncias com até 35 clientes e mais de 100 itens.

Para lidar com instâncias de maior escala, Gendreau et al. (2008) propuseram a primeira abordagem de solução meta-heurística da literatura, que emprega Tabu Search (TS) para otimizar as características de roteirização, enquanto os padrões de carregamento viáveis são identificados por meio de um procedimento heurístico baseado na heurística Touching Perimeter (TP) de Lodi et al. (1999). Os autores criaram um conjunto de 20 novas instâncias do 2L-CVRP. Tais instâncias, juntamente com as de Iori et al. (2007) constituem as instâncias de referência do 2L-CVRP. Todas as instâncias estão disponíveis no site http://www.or.deis.unibo.it/research.html.

Fuellerer et al. (2009) empregaram várias técnicas de empacotamento bidimensional (Bottom-Left Fill - BLF, Touching Perimeter Algorithm - TP) para encontrar padrões de carregamento viáveis, combinadas com a metaheurística Ant Colony Optimization (ACO) para a otimização global. Este foi o primeiro trabalho em que o 2L-CVRP com carregamento não orientado foi abordado e no qual as quatro versões do problema foram avaliadas. Uma contribuição interessante deste artigo reside na avaliação do efeito das restrições de carregamento sobre os custos totais.

Zachariadis et al. (2009) apresentaram a meta-heurística Guided Tabu Search (GTS) para solucionar os aspectos de roteamento do problema e cinco heurísticas de empacotamento para resolver os aspectos de carregamento: BottomLeft Fill (W-axis) - BLF-W; Bottom-Left Fill (L-axis) BLF-L; Max Touching Perimeter heuristic - MTP; Max Touching Perimeter No Walls heuristic - MTPNW e Min Area heuristic - MA. Os autores testaram a abordagem de solução no conjunto de instâncias de referência.

Azevedo et al. (2009) apresentaram um algoritmo B\&C para o 2L-CVRP, sendo esta a segunda abordagem exata encontrada na literatura. Este trabalho difere-se do de Iori et al. (2007) pelo uso de diferentes famílias de desigualdades válidas para o CVRP. Para analisar a viabilidade dos carregamentos utilizaram-se os seguintes algoritmos heurístico e exato, respectivamente: Bottom Left Decreasing Width heuristic - BLDW de Baker et al. (1980) e OneBin Algorithm de Martello et al. (2000). Os resultados para o

Tabela 1. Classes usadas para geração de itens no 2L-CVRP Fonte: lori et al. (2007)

\begin{tabular}{|c|c|c|c|c|c|c|c|}
\hline \multirow[b]{2}{*}{ Classe } & \multirow[b]{2}{*}{$m_{i}$} & \multicolumn{2}{|c|}{ Vertical } & \multicolumn{2}{|c|}{ Homogêneo } & \multicolumn{2}{|c|}{ Horizontal } \\
\hline & & $l_{i p}$ & $w_{i p}$ & $l_{i p}$ & $w_{i p}$ & $l_{i p}$ & $w_{i p}$ \\
\hline 1 & {$[1,1]$} & {$[1,1]$} & {$[1,1]$} & {$[1,1]$} & {$[1,1]$} & {$[1,1]$} & {$[1,1]$} \\
\hline 2 & {$[1,2]$} & $\frac{4 L}{10}, \frac{9 L}{10}$ & {$\left[\frac{W}{10}, \frac{2 W}{10}\right]$} & {$\left[\frac{2 L}{10}, \frac{5 L}{10}\right]$} & {$\left[\frac{2 W}{10}, \frac{5 W}{10}\right]$} & $\frac{L}{10}, \frac{2 L}{10}$ & {$\left[\frac{4 W}{10}, \frac{9 W}{10}\right.$} \\
\hline 3 & {$[1,3]$} & $\frac{3 L}{10}, \frac{8 L}{10}$ & {$\left[\frac{W}{10}, \frac{2 W}{10}\right]$} & {$\left[\frac{2 L}{10}, \frac{4 L}{10}\right]$} & {$\left[\frac{2 W}{10}, \frac{4 W}{10}\right]$} & $\frac{L}{10}, \frac{2 L}{10}$ & {$\left[\frac{3 W}{10}, \frac{8 W}{10}\right]$} \\
\hline 4 & {$[1,4]$} & $\frac{2 L}{10}, \frac{7 L}{10}$ & {$\left[\frac{W}{10}, \frac{2 W}{10}\right]$} & {$\left[\frac{L}{10}, \frac{4 L}{10}\right]$} & {$\left[\frac{W}{10}, \frac{4 W}{10}\right]$} & $\frac{L}{10}, \frac{2 L}{10}$ & {$\left[\frac{2 W}{10}, \frac{7 W}{10}\right]$} \\
\hline 5 & {$[1,5]$} & {$\left[\frac{L}{10}, \frac{6 L}{10}\right]$} & {$\left[\frac{W}{10}, \frac{2 W}{10}\right]$} & {$\left[\frac{L}{10}, \frac{3 L}{10}\right]$} & {$\left[\frac{L}{10}, \frac{3 L}{10}\right]$} & $\frac{L}{10}, \frac{2 L}{10}$ & {$\left[\frac{W}{10}, \frac{6 W}{10}\right]$} \\
\hline
\end{tabular}


caso 2|SO|L mostraram redução do tempo computacional quando comparados com os valores gerados pelo método exato de Iori et al. (2007).

Strodl et al. (2010) utilizaram a meta-heurística $V a$ riable Neighborhood Search (VNS) para solucionar a parte de roteamento do $2 \mathrm{~L}-\mathrm{CVRP}$, incorporando um procedimento heurístico e outro exato para resolver o problema de empacotamento. $\mathrm{O}$ aspecto de empacotamento do problema é solucionado em um procedimento de três passos. O primeiro passo consiste em verificar se as restrições de capacidade máxima do veículo em termos de peso e área ocupada são satisfeitas. O segundo passo aplica a heurística TP. Se a heurística não encontra uma solução viável, então o terceiro passo, que consiste no método exato $\mathrm{B} \& \mathrm{~B}$, é executado. A meta-heurística desenvolvida foi testada nas instâncias de referência e os resultados foram comparados com o ACO de Fuellerer et al. (2009).

Leung et al. (2010) apresentaram uma meta-heurística Simulated Annealing (SA), ao mesmo tempo em que usaram um conjunto de heurísticas de empacotamento para solucionar a componente de carregamento do problema: as cinco propostas por Zachariadis et al. (2009) mais a heurística Max Fitness Value Heuristic (MFVH). Os autores compararam seus resultados com aqueles obtidos por Gendrau et. al. (2008), por Zachariadis et al. (2009) e por Fuellerer et al. (2009). Para o 2L-CVRP irrestrito, o SA apresentou ganhos em relação ao TS de Gendrau et. al. (2008) e ao GTS de Zachariadis et al. (2009). Quanto ao ACO de Fuellerer et al. (2009), teve-se um desempenho melhor apenas para a versão sequencial do problema.

Leung et al. (2011) propuseram uma meta-heurística Extended Guided Tabu Search (EGTS), para a roteirização do problema. Para resolver a componente de empacotamento do problema, os autores acrescentaram a heurística $\mathrm{H}_{6}$ - Lowest Reference Line Best-Fit heuristic (LBFH) às heurísticas propostas por Zachariadis et al. (2009). O método foi testado e apresentou melhores resultados em relação ao GTS de Zachariadis et al. (2009).

Duhamel et al. (2011) propuseram uma meta-heurística Greedy Randomized Adaptive Search Procedure (GRASP) combinada com Evolutionary Local Search (ELS) para solucionar o 2L-CVRP. Em comparação com Gendrau et al. (2008), Zachariadis et al. (2009) e Fuellerer et al. (2009), a meta-heurística proposta conseguiu obter melhor desempenho tanto em termos de redução de custo quanto de tempo de processamento.

Shen e Murata (2012) resolveram o 2L-CVRP usando Genetic Algorithm (GA). Eles consideraram que o veículo sai vazio do depósito e coleta os itens dos clientes ao longo da rota. Os autores não efetuaram comparações com resultados já publicados na literatura do 2L-CVRP.

Zachariadis et al. (2013) propuseram a meta-heurística Promise Routing-Memory Packing (PRMP). Os autores testaram a meta-heurística usando as instâncias de referência, e compararam os resultados com a melhor solução obtida por Leung et al. (2011), por Fuellerer et al. (2009) e por Duhamel et al. (2011) e obtiveram uma melhoria na solução encontrada.

Bin et al. (2013) apresentaram a meta-heurística Artificial Bee Colony Algorithm (ABC) e três heurísticas de empacotamento (BLF, MTP e LBFH) para resolver o 2L-
CVRP. A meta-heurística foi testada em 10 das instâncias de referência, englobando até 100 clientes e 310 itens. Os resultados do ABC foram comparados com as meta-heurísticas de Gendrau et al. (2008), Zachariadis et al. (2009) e Leung et al. (2011), obtendo melhorias em relação a todos.

Dominguez et al. (2014a) apresentaram um modelo de Programação Linear Inteira (PLI) para o 2L-CVRP e propuseram uma meta-heurística Multistart Biased-Randomized Algorithm (MS-BR). Esta foi a primeira abordagem da literatura que integrou o processo de empacotamento como parte do procedimento de construção da rota. Os resultados foram comparados com Fuellerer et al. (2009), mostrando um desempenho melhor. Já para a versão 2|UO|L comparou-se com os resultados de Fuellerer et al. (2009), Leung et al. (2010), Leung et al. (2011) e Duhamel et al. (2011), e verificaram-se os menores gaps médios em relação às melhores soluções.

Abdal-Hammed et al. (2014) propuseram a metaheurítica Large Neighborhood Search (LNS) para resolver a versão 2|UO|L do 2L-CVRP. O LNS é aplicado tanto para resolver o aspecto de roteirização quanto o de carregamento. Os resultados foram comparados com aqueles obtidos pelo GRASPxELS de Duhamel et al. (2011) para as classes de 2 a 5. Segundo os resultados numéricos, o LNS apresentou um melhor desempenho em cerca de $20 \%$ dos casos avaliados em relação à solução obtida por Duhamel et al. (2011).

Wei et al. (2015) desenvolveram uma meta-heurística Variable Neighborhood Search (VNS) para tratar o aspecto de roteirização e incorporaram uma heurística Skyline adaptada a fim de resolver a componente de carregamento do 2L-CVRP. Foram efetuados experimentos computacionais utilizando as instâncias de referência para solucionar as versões 2|UO|L e 2|SO|L do problema. A solução obtida pelo VNS foi comparada com os melhores valores obtidos entre Fuellerer et al. (2009), Leung et al. (2010), Leung et al. (2011), Duhamel et al. (2011) e Zachariadis et al. (2013). O VNS alcançou melhorias em relação às melhores soluções conhecidas de 0,05 a $0,53 \%$ para a versão $2|\mathrm{UO}| \mathrm{L}$ e de no mínimo de $0,58 \%$ para a versão $2|\mathrm{SO}| \mathrm{L}$. A metaheurística proposta encontrou melhores soluções para 65 instâncias do 2L-CVRP irrestrito e 88 instâncias do 2LCVRP sequencial de um total de 144.

Algumas variantes do 2L-CVRP também têm sido analisadas na literatura recente, que incluem desde a incorporação de variações clássicas relevantes do VRP, como coleta e entrega, janelas de tempo e frota heterogênea, assim como diversificações em relação ao aspecto de carregamento.

Malapert et al. (2008) apresentaram um modelo para o Two-Dimensional Pickup and Delivery Routing Problem with Loading Constraints (2L-PDP). No entanto, os autores não apresentaram testes com instâncias de referência e não obtiveram sucesso ao aplicar a heurística BLF.

Logo, o problema com coleta e entrega não foi solucionado. Contudo, inclui-se este artigo nesta revisão pois foi a primeira abordagem a incluir coleta e entrega e, mesmo que não sucedida, demonstrou uma direção de pesquisa que pode ser seguida. 


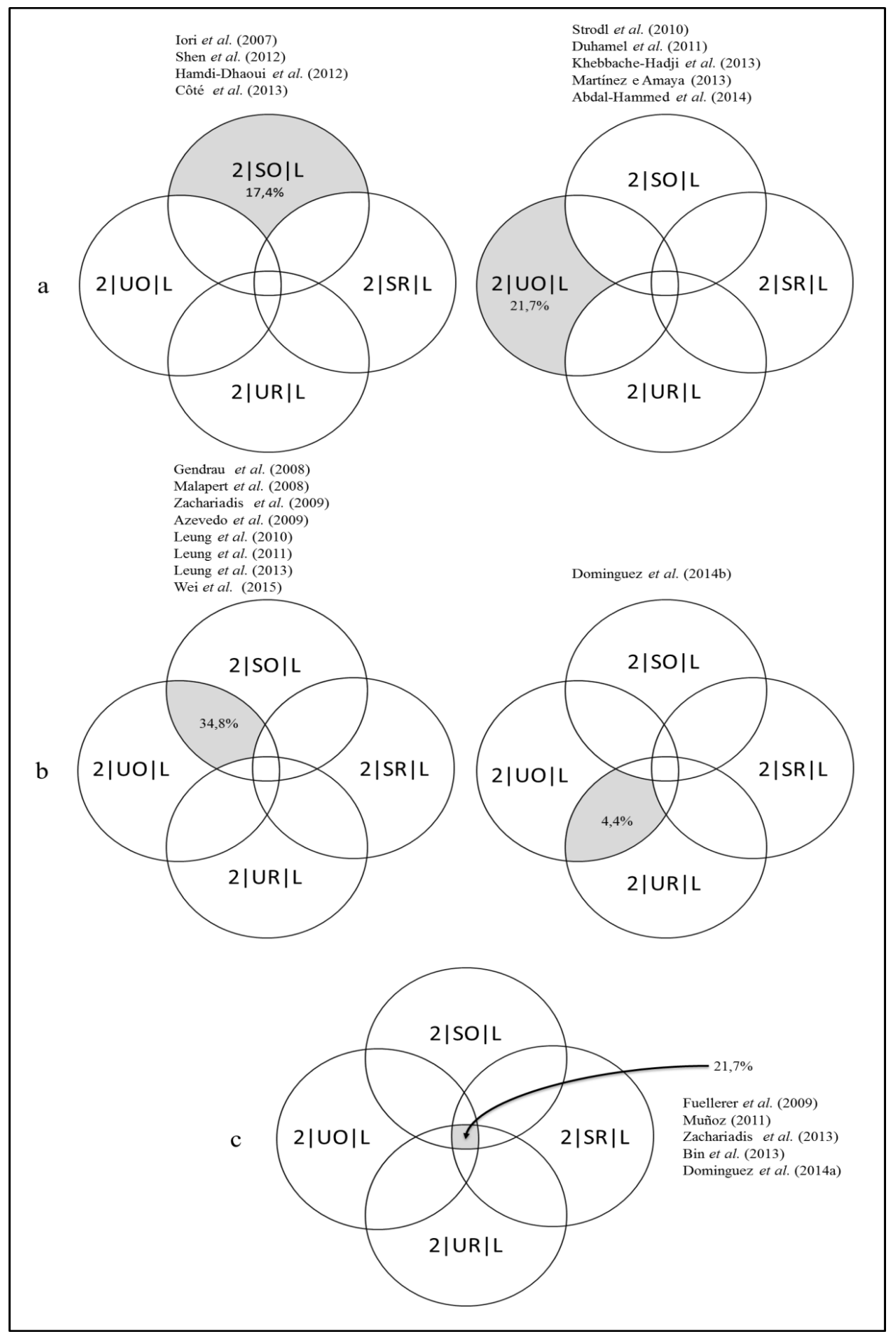

Figura 2: (a) Distribuição de artigos que estudam apenas uma categoria do 2L-CVRP; (b) Distribuição de artigos que estudam duas categorias simultaneamente do 2L-CVRP; (c) Distribuição de artigos que estudam quatro categorias do 2L-CVRP

Muñoz (2011) apresentou uma nova modelagem para o 2L-CVRP, denominada Capacitated Vehicle Routing Problem with Two-dimensional Loading Constraints and Handling Costs (2L-CVRP-H), cuja função objetivo minimiza, além dos custos de roteirização, os custos de manipular os itens no local de demanda.

Hamdi-Dhaoui et al. (2012) propuseram uma metaheurística Nondominated Sorting Genetic Algorithm II (NSGA -II) para resolver o Two-dimensional Loading Capacitated Vehicle Routing Problem with Partial Conflicts (2LPC-CVRP). Neste caso, os autores realizaram um estudo bi-objetivo, dentro do qual se considerou a minimização do custo de transporte bem como o balanceamento de carga entre as rotas, minimizando a diferença em termos de superfície ocupada entre a rota mais carregada e a rota menos carregada. Para os aspectos de empacotamento foi aplicada a heurística BLF adaptada. Foi o primeiro artigo a abordar balanceamento de carga.

Leung et al. (2013) abordaram o Two-Dimensional Loading Heterogeneous Fleet Vehicle Routing Problem (2L-HFVRP). Foi proposta uma meta-heurística Simulated Annealing with Heuristic Local Search (SA_HLS). O desempenho do SA_HLS foi testado em instâncias de referência do 2L-CVRP para as versões 2|SO|L e 2|UO|L e os resultados foram comparados com as meta-heurísticas propostas por Zachariadis et al. (2009), Fuellerer et al. (2009) e Duhamel et al. (2011). Obteve-se o melhor desempenho médio somente em relação ao GTS de Zachariadis et al. (2009) para ambas as versões. 
Tabela 2. Resumo da revisão da literatura de $2 \mathrm{~L}-\mathrm{CVRP}$

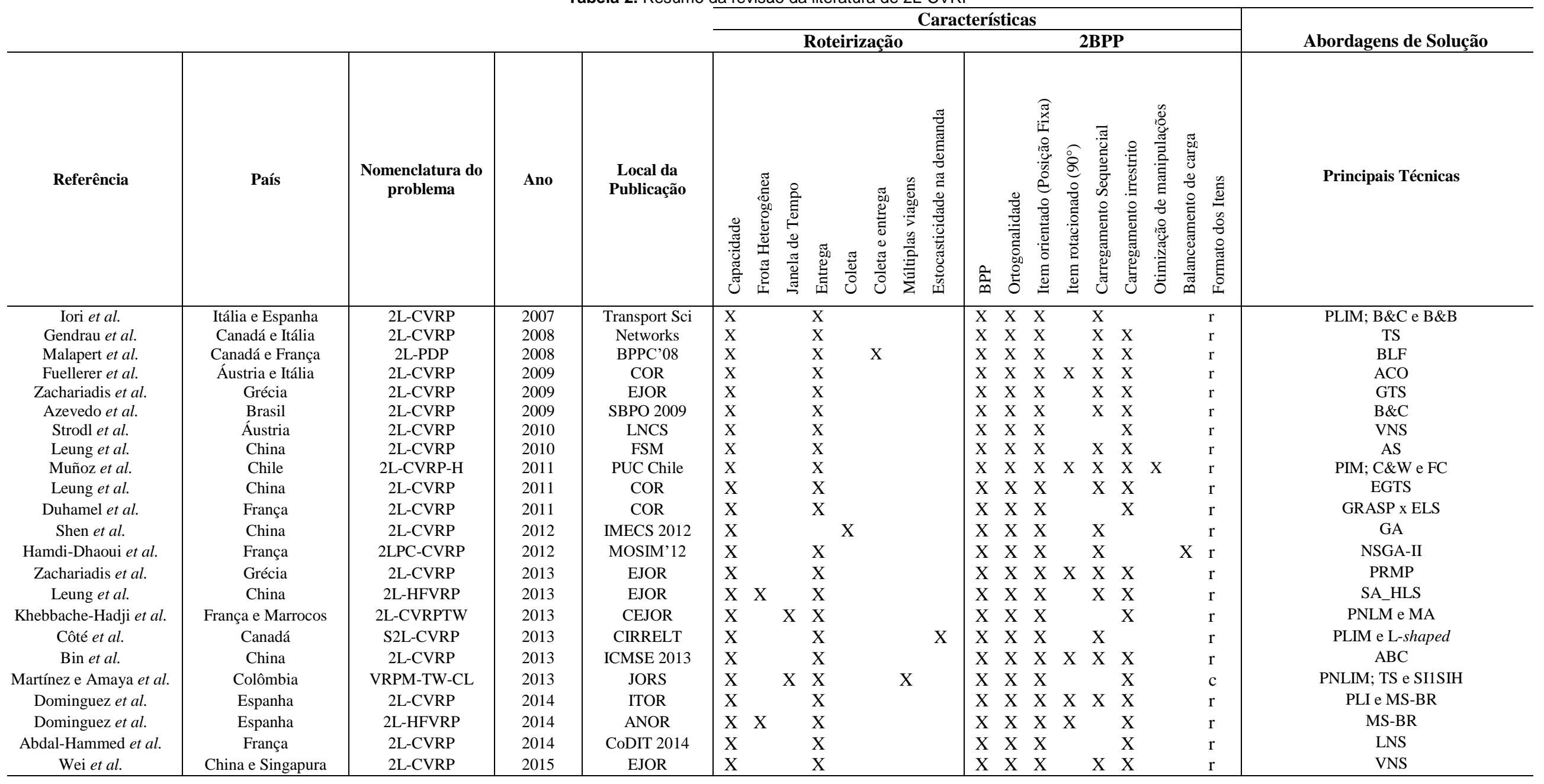


Khebbache-Hadji et al. (2013) trataram o Two-Dimensional Loading Capacitated Vehicle Routing Problem with Time Windows (2L-CVRPTW). Os autores apresentaram um modelo de Programação Não-Linear Inteira Mista e propuseram seis heurísticas, advindas da combinação de duas heurísticas para solucionar o Vehicle Routing Problem with Time Windows (VRPTW) e três para empacotar itens dentro de veículo, além da meta-heurística Memetic Algorithm (MA) para a resolução do 2L-CVRPTW.

Côté et al. (2013) abordaram o Vehicle Routing with Stochastic Two-Dimensional Items (S2L-CVRP) e propuseram uma modelagem matemática, cuja função objetivo é minimizar os custos de roteirização e os custos recursivos esperados. Para a resolução, utilizou-se o método $L$-shaped inteiro de Laporte e Louveaux (1993). O método foi testado, via CPLEX 12.5, nas instâncias de referência para o caso determinístico, solucionando instâncias com até 71 clientes e 226 itens. Já para o caso estocástico, com base nas instâncias supracitadas, os autores geraram instâncias específicas, resolvendo dentro do tempo de duas horas instâncias com até 44 clientes.

Martínez e Amaya (2013) apresentaram o Vehicle Routing Problem With Multi-Trips, Time Windows And Two-Dimensional Circular Loading Constraints (VRPMTW-CL), que considera restrições para lidar com itens circulares. Este foi o primeiro estudo de caso da literatura e, para resolver problemas de tamanho real, os autores propuseram uma heurística de duas etapas que usa Solomon I1 Sequential Insertion Heuristic, (SI1SIH) e TS.

Dominguez et al. (2014b) resolveram o 2L-HFVRP considerando a possibilidade de rotacionar os itens. Para isso, os autores utilizaram o mesmo algoritmo híbrido proposto em Dominguez et al. (2014a), o MS-BR, com a adição da heurística TP para resolver o aspecto de carregamento. O MS-BR foi testado nas instâncias de Leung et al. (2013). Ao comparar os cenários com e sem rotação resol- vidos pelo MS-BR, pode-se comprovar que, como era esperado, os cenários que permitiam a rotação dos itens viabilizaram redução nos custos totais.

A Tabela 2 sumariza, em ordem cronológica, a revisão da literatura, destacando as principais características do 2L-CVRP e suas variantes tratadas pelos pesquisadores, bem como a principal abordagem de resolução utilizada. $\mathrm{Na}$ tabela, a característica "Formato dos Itens" é simbolizada pelas siglas $r$, que designa o formato retangular, e $c$, que indica forma circular.

A Figura 2 mostra a distribuição dos artigos conforme as categorias do 2L-CVRP.

Na Figura 2a observa-se que 39,1\% dos artigos exploraram apenas uma das categorias, sendo que $17,4 \%$ tratam do 2|SO|L e 21,7\% do 2|UO|L. Quanto ao estudo de duas categorias simultaneamente, nota-se pela Figura $2 \mathrm{~b}$ que a grande maioria dos artigos abordou as versões orientadas $(2|\mathrm{SO}| \mathrm{L}$ e $2|\mathrm{UO}| \mathrm{L})$, correspondendo à $34,8 \%$ do total, enquanto há apenas um artigo, que equivale a 4,4\% do total, que engloba as duas classes irrestritas do problema $(2|\mathrm{UO}| \mathrm{L}$ e $2|\mathrm{UR}| \mathrm{L})$.

A Figura 2c mostra que os artigos que avaliam as quatro categorias do problema ao mesmo tempo correspondem a 21,7\% do total. Entre estes, somente o de Muñoz (2011) estuda uma variante do 2L-CVRP.

A Tabela 3 apresenta as características do ambiente de desenvolvimento e de teste empregadas em cada artigo para resolução dos modelos e das meta-heurísticas propostos.

A partir da Tabela 3 nota-se que a linguagem de programação mais utilizada foi o $\mathrm{C}++$, correspondendo a $40,9 \%$ do total, e o solver mais usado foi o CPLEX, representando $57 \%$ entre os sete que usaram software de apoio.

Tabela 3. Características do Ambiente de Desenvolvimento e de Testes

\begin{tabular}{|c|c|c|c|c|}
\hline Referência & $\begin{array}{l}\text { Linguagem e } \\
\text { Programação }\end{array}$ & $\begin{array}{c}\text { Sistema } \\
\text { Operacional }\end{array}$ & $\begin{array}{l}\text { Características do } \\
\text { Computador }\end{array}$ & $\begin{array}{l}\text { Software de apoio para } \\
\text { resolver MIP, MILP }\end{array}$ \\
\hline Iori et al. & $\mathrm{C}$ & $?$ & Pentium IV, $3 \mathrm{GHz}$ & CPLEX 9.0 \\
\hline Gendrau et al. & $\mathrm{C}$ & ? & Pentium IV, $1.7 \mathrm{GHz}$ & ? \\
\hline Malapert et al. & $?$ & $?$ & ? & $?$ \\
\hline Fuellerer et al. & ANSI C++ & Linux & Pentium IV, $3.2 \mathrm{GHz}$ & $?$ \\
\hline Zachariadis et al. & Visual $\mathrm{C}++$ & Windows XP & Pentium IV, $2.4 \mathrm{GHz}$ & $?$ \\
\hline Azevedo et al. & $\mathrm{C}$ & ? & Intel Quad Core, $2.4 \mathrm{GHz}$ & X-PRESS-OPTIMIZER \\
\hline Strodl et al. & $\mathrm{C}++$ & Linux & Quad Core & ? \\
\hline Leung et al. & $\mathrm{C}++$ & Windows XP & Core Duo, $2.4 \mathrm{GHz}$ & $?$ \\
\hline Muñoz et al. & $\mathrm{C}++$ & ? & Intel Core ${ }^{\mathrm{TM}} \mathrm{i} 7,2.67 \mathrm{GHz}$ & CPLEX 12.0 \\
\hline Leung et al. & ANSI C++ & Windows XP & Core 2 Duo, $2.0 \mathrm{GHz}$ & ? \\
\hline Duhamel et al. & $\mathrm{C}++$ & Linux & Opteron, $2.1 \mathrm{GHz}$ & CPLEX 11.0 \\
\hline Shen et al. & Java & Windows XP & Core $^{\mathrm{TM}} 2 \mathrm{Duo}, 2.53 \mathrm{GHz}$ & LINGO \\
\hline Hamdi-Dhaoui et al. & $\mathrm{C}++$ & Windows 7 & Pentium Dual Core, $2.2 \mathrm{GHz}$ & $?$ \\
\hline Zachariadis et al. & Visual $\mathrm{C}++$ & ? & Intel E6600, 2.4GHz & $?$ \\
\hline Leung et al. & $\mathrm{C}++$ & Windows 7 & Core $^{\mathrm{TM}} 2 \mathrm{Duo}, 2.2 \mathrm{GHz}$ & $?$ \\
\hline Khebbache-Hadji et al. & Delphi & Windows XP & Pentium IV, $3.6 \mathrm{GHz}$ & $?$ \\
\hline Côté et al. & $\mathrm{C}++$ & Linux & Intel Xeon X5675, 3.07GHz & CPLEX 12.5 \\
\hline Bin et al. & Visual C++ & Windows 7 & Core 2 i5, $2.5 \mathrm{GHz}$ & ? \\
\hline Martínez e Amaya et al. & Visual Basic & $?$ & Intel Xeon, $1.86 \mathrm{GHz}$ & X-PRESS IVE \\
\hline Dominguez et al. & Java & Windows 7 & Intel Core ${ }^{\mathrm{TM}} 2$ Duo, $2.4 \mathrm{GHz}$ & $?$ \\
\hline Dominguez et al. & Java & Windows 7 & Intel Core ${ }^{\mathrm{TM}}$ i7, $2.2 \mathrm{GHz}$ & $?$ \\
\hline Abdal-Hammed et al. & $\mathrm{C}++$ & $?$ & $\begin{array}{l}\text { Pentium Core i5-2500, } 3.3 \\
\text { GHz }\end{array}$ & $?$ \\
\hline Wei et al. & $\mathrm{C}++$ & Linux & Intel Xeon E5430, $2.66 \mathrm{GHz}$ & ? \\
\hline
\end{tabular}




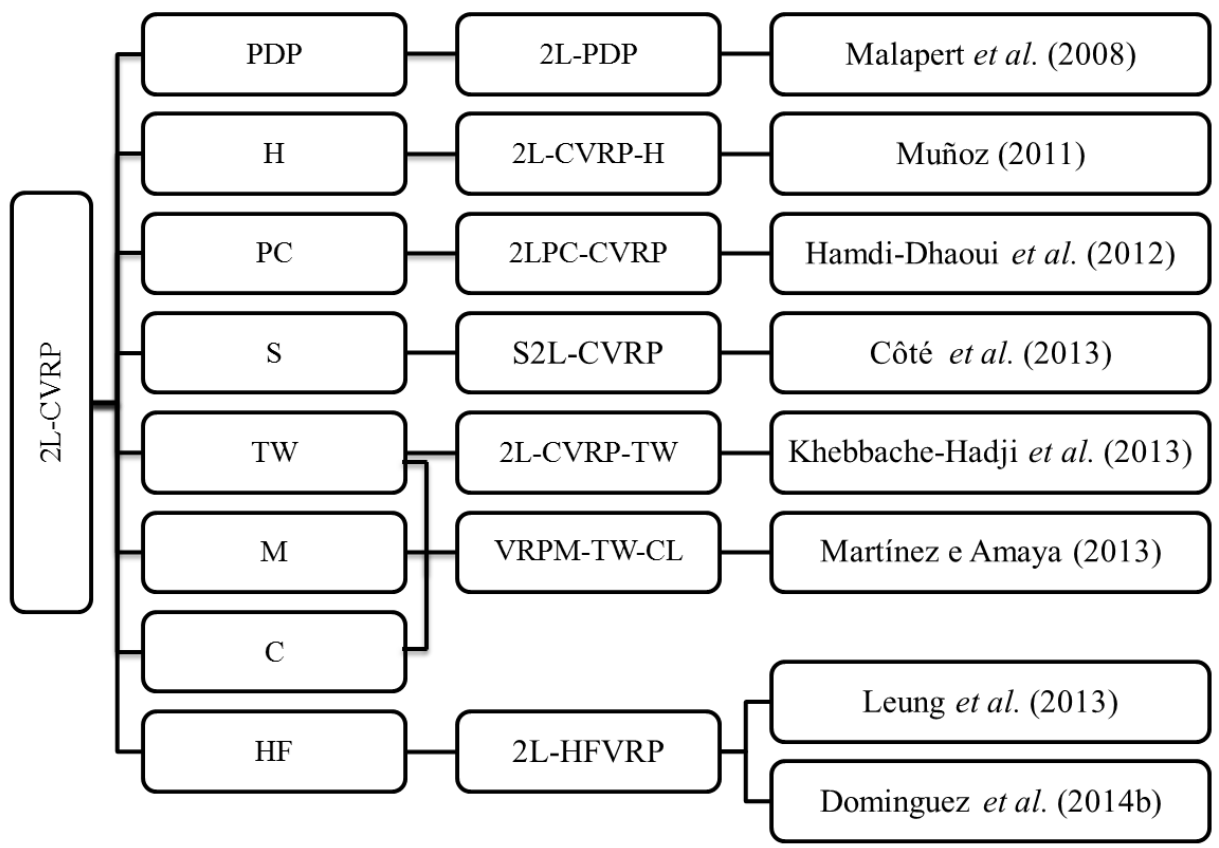

Legendas:

PDP: Pickup and Delivery Problem

S: Stocastic

PC: Partial Conflict
M: Multitrips
H: Handling Costs
TW: Time Windows

HF: Heterogeneos Fleet

Figura 3. Variantes do 2L-CVRP

\subsection{Fase III: Documentação da Revisão}

Nesta subseção será apresentada apenas a etapa 7 da Fase III, já que a etapa 8 representa a própria elaboração do artigo.

\subsubsection{Etapa 7: Analisar Resultados}

A Tabela 2 evidencia que entre os 23 artigos revisados, foram elaborados ao todo sete modelos matemáticos, que foram estruturados como programação linear e não linear inteira mista. Em termos de abordagens exatas, Iori et al. (2007) e Azevedo et al.(2009) empregaram uma técnica similar (B\&C) para resolver o problema, enquanto Muñoz (2011), Shen et al. (2012), Côté et al. (2013), Martínez e Amaya (2013) solucionaram instâncias de pequena escala de modo exato com o apoio de softwares como CPLEX, LINGO e X-PRESS. Nota-se que $87 \%$ dos autores utilizaram métodos heurísticos e metaheurísticos para construir uma solução para o 2L-CVRP. Isso se deve à necessidade de alcançar soluções para instâncias de escalas maiores, que são mais condizentes com os problemas práticos. Apenas seis dos 23 artigos analisados incluem a rotação de $90^{\circ}$ dos itens.

Com exceção do artigo de Dominguez et al. (2014a,b), que propuseram uma abordagem integrada de resolução, em geral, os métodos de solução se baseiam em uma estrutura similar: a componente de roteirização consiste no problema principal do algoritmo que constrói soluções e, iterativamente, chama algum algoritmo de empacotamento (subproblema), o qual verifica se a solução gerada é viável em termos de restrições de carregamento. A solução inicial, usualmente, é obtida via algoritmos construtivos, como, por exemplo, a heurística de Clarke e Wright (1964), que foi utilizada em alguns dos artigos analisados. Após execução da parte construtiva, normalmente, incorporam-se algoritmos de melhoria.
Além disso, observa-se que na literatura há poucas variantes do 2L-CVRP, sendo que os trabalhos desse gênero representam $34,8 \%$ do total analisado. Nesse universo, o artigo de Martínez e Amaya (2013) é o único que incorpora mais de uma variante (janela de tempo, múltiplas viagens e itens circulares). Os demais autores tratam de apenas uma variante, que neste caso são: janela de tempo, frota heterogênea, coleta e entrega, otimização de manipulações, balanceamento de carga e estocasticidade da demanda, existindo apenas um artigo para cada uma dessas características, com exceção de frota heterogênea, para o qual há dois artigos (Figura 3).

É válido destacar que existe uma tendência de crescimento para pesquisas com enfoque em diversificações do problema, o que é ressaltado pelo fato de que cinco das oito publicações com variações foram publicadas a partir de 2013.

Em suma, pode-se afirmar que há uma constante busca por novos métodos de resolução, que garantam um tempo de execução mais rápido e uma solução mais próxima da otimalidade. E também existe uma maior propensão para tornar o problema mais realista e aderente às necessidades práticas incorporando variantes ao modelo original.

Apesar dos avanços percebidos nas pesquisas de 2013 e 2015, há ainda muitas lacunas a serem preenchidas nesta área, como, por exemplo: Explorar problemas com aplicação real (estudos de caso); Solucionar o problema com Coleta e Entrega, pois o mesmo foi proposto, porém não resolvido; Fazer mais combinações entre as variantes do problema de VRP, como por exemplo, incorporar frota heterogênea com coleta e entrega, janelas de tempo e estocasticidade da demanda ao mesmo tempo; Criar mais modelos matemáticos que representem a incorporação das variantes; Explorar abordagens de resolução exatas que consigam solucionar instâncias de grande escala; Todos os pro- 
blemas tratados se baseiam no modo de transporte rodoviário, via caminhão. É necessário explorar casos com aplicações em outros modos, como aquaviário, com ênfase no transporte por navios, e o modo aéreo; Adicionar restrições de equilíbrio de carga; Explorar mais funções multiobjetivo que englobem as necessidades do mundo real.

\section{CONSIDERAÇÕES FINAIS}

Neste artigo apresentou-se uma revisão sistemática da literatura para o 2L-CVRP e suas variantes, enfatizando, sobretudo, os métodos de resolução utilizados pelos autores, que consistem em um dos principais fatores de diferenciação entre as publicações. Esta área de pesquisa, apesar de recente, tem conquistado a atenção da comunidade científica pela importância que o problema representa tanto em termos teóricos quanto práticos.

Entre as abordagens de solução propostas, pode-se afirmar que a grande maioria é composta por heurísticas e meta-heurísticas, o que pode ser explicado pela necessidade de se alcançar soluções de problemas de tamanho real. Também é possível notar que tem aumentado a quantidade de pesquisas que incluem variantes clássicas do VRP, como, por exemplo, janela de tempo e frota heterogênea.

\section{AGRADECIMENTOS}

Os autores agradecem à CAPES, à FAPES (75528452) e ao CNPq pelo apoio financeiro.

\section{REFERÊNCIAS}

Abdal-Hammed, M.K.; Hifi, M.; Wu; L. (2014) Large neighborhood search for the vehicle routing problem with twodimensional loading constraints. In: International Conference on Control, Decision and Information Technologies (CoDIT). IEEE, p. 054-059.

Araujo, R. R. de. (2010) Uma abordagem de resolução integrada para os problemas de roteirização e carregamento de veículos. Tese Doutorado, Programa de Pós-Graduação em Engenharia de Produção, Universidade Federal do Rio Grande do Sul, Porto Alegre.

Azevedo, B. L. P. ; Hokama, P. H.; Miyazawa, F. K.; Xavier, E. C. (2009) A branch-and-cut approach for the vehicle routing problem with two-dimensional loading constraints. In: Simpósio Brasileiro de Pesquisa Operacional, v. 41.

Baker, B. S.; Coffman Jr., E. G.; Rivest, R. L. Orthogonal Packings In Two Dimensions. Siam Journal On Computing, v. 9 n. 4, p. 846-855, 1980.

Bin, W.; Hong, C.; Zhi-Yong, C. (2013) Artificial bee colony algorithm for two-dimensional loading capacitated vehicle routing problem. In: International Conference on Management Science and Engineering (ICMSE), IEEE, p. 406-412.

Brereton, P.; B. A. Kitchenham; D. Budgen; M. Turner e M. Khalil (2007) Lessons from applying the systematic literature review process within the software engineering domain. The Journal of Systems and Software, v. 80, p. 571-583.

Côté, J.-F.; Gendreau, M.; Potvin, J.-Y. (2013) The Vehicle Routing Problem with Stochastic Two-Dimensional Items, CIRRELT-2013-84.
Clarke, G., Wright, J.W. (1964) Scheduling of Vehicles from a Central Depot to a Number of Delivery Points. Operations Research, v. 12, p. 568-581.

Dominguez, O.; Juan, A. A.; Faulin, J. A. (2014a) Biasedrandomized algorithm for the two-dimensional vehicle routing problem with and without item rotations. Intern. Transactions in Operational Research, p. 1-14.

Dominguez, O.; Juan, A. A.; Barrios, B.; Faulin, J.; Agustin, A. (2014b) Using biased randomization for solving the twodimensional loading vehicle routing problem with heterogeneous fleet. Annals of Operations Research, p. 1-22.

Duhamel, C.; Lacomme, P.; Quilliot, A.; Toussaint, H. (2011) A multi-start evolutionary local search for the two-dimensional loading capacitated vehicle routing problem. Computers \& Operations Research, v. 38, n. 3, p. 617-640.

Fuellerer, G.; Doerner, K. F.; Hartl, R. F.; Iori, M. (2009) Ant colony optimization for the two-dimensional loading vehicle routing problem. Computers \& Operations Research, v. 36, p. $655-673$.

Gendreau, M.; Iori, M.; Laporte, G.; Martello, S. (2008) A Tabu Search heuristic for the vehicle routing problem with twodimensional loading constraints. Networks, v. 51, n. 1, p. 4-18.

Hamdi-Dhaoui, K. ; Labadie, N.; Yalaoui, A. (2012) Problème de tournées de véhicules avec chargement bidimensionnel et contraintes de conflits partiels multi-objectif. Proceedings of MOSIM'12.

Iori, M.; Gonzáles, J. J. S.; Vigo, D. (2007) An exact approach for the vehicle routing problem with two-dimensional loading constraints. Transportation Science, v. 41 (2), p. 253-264.

Iori, M.; Martello, S. (2010) Routing problems with loading constraints. Top, v. 18, n. 1, p. 4-27.

Iori, M.; Martello, S. (2013) An annotated bibliography of combined routing and loading problems. The Yugoslav Journal of Operations Research. ISSN: 0354-0243 EISSN: 2334-6043, v. 23 , n. 3.

Khebbache-hadji, S. ; Prins, C.; Yalaoui, A.; Reghioui, M. (2013) Heuristics and memetic algorithm for the twodimensional loading capacitated vehicle routing problem with time windows. Central European Journal of Operations Research, v. 21, n. 2, p. 307-336.

Laporte, G.; Louveaux, F. V. (1993) The integer L-shaped method for stochastic integer programs with complete recourse. Operations research letters, v. 13, n. 3, p. 133-142.

Labadie, N.; Prins, C. (2012) Vehicle Routing Nowadays: Compact Review and Emerging Problems. In: Mejía, G.; Velasco, N. (Ed.). Production systems and supply chain management in emerging countries: best practices, Springer, $\mathrm{p}$. 141-166.

Leung, S. C. H.; Zheng, J.; Zhang, D.; Zhou, X.(2010) Simulated annealing for the vehicle routing problem with twodimensional loading constraints. Flexible services and manufacturing journal,v.22, p. 61-82.

Leung, S. C. H.; Zhou, X.; Zhang, D.; Zheng, J. (2011) Extended guided tabu search and a new packing algorithm for the two-dimensional loading vehicle routing problem. Computers \& Operations Research, v. 38, p. $205-215$. 
Leung, S. C. H.; Zhang, Z..; Zhang, D.; Hua, X.; Lim, M. K. (2013) A meta-heuristic algorithm for heterogeneous fleet vehicle routing problems with two-dimensional loading constraints. European Journal of Operational Research, v. 225, p. $199-210$.

Lodi, A.; Martello, S.; Vigo, D. (1999) Heuristic and metaheuristic approaches for a class of two-dimensional bin packing problems. INFORMS Journal on Computing, v. 11, n. 4, p. 345-357.

Malapert, A.; Guerét, C.; Jussien, N.; Langevin, A.; Rousseau, L.-M. (2008) Two-dimensional pickup and delivery routing problem with loading constraints. In Proceedings of the First CPAIOR Workshop on Bin Packing and Placement Constraints (BPPC'08), France.

Martello, S.; Pisinger, D.; Vigo, D. (2000) The Three-

Dimensional Bin Packing Problem. Operations Research, V. 48, N. 2, P. 256-267.

Martínez, L.; Amaya, C. A. (2013) A vehicle routing problem with multi-trips and time windows for circular items. Journal of the Operational Research Society, v. 64, n. 11, p. 1630-1643.

Muñoz, A. L. (2011) Solución al problema de ruteo de vehículos con restricciones de capacidad y reordenamiento de carga en los sitios de demanda. $84 \mathrm{f}$. Tesis Doutorado, PUC de Chile, Chile.

Okoli, C. e K. Schabram (2010) A Guide to Conducting a Systematic Literature Review of Information Systems Research. Sprouts: Working Papers on Information Systems, v. 10, n. 26.

Ranck Júnior, R.; Yanasse, H. H. (2010) O Problema Integrado de Carregamento e Roteamento de Veículos: uma revisão parcial dos trabalhos da literatura. In: $X$ Worcap. Instituto Nacional de Pesquisas Espaciais - INPE: São José dos Campos.

Shen, Y.; Murata, T. (2012) Pick-up Scheduling of Twodimensional Loading in Vehicle Routing Problem by using GA. In: Proceedings of the International MultiConference of Engineers and Computer Scientists, IMECS, v. 2, p. 1532-1537, Hong Kong.

Strodl, J.; Doerner, K.F.; Tricoire, F.; Hartl, R.F. (2010) On index structures in hybrid metaheuristics for routing problems with hard feasibility checks: An application to the 2-dimensional loading vehicle routing problem. In: Blesa, M.J.; Blum, C.; Raidl, G.; Roli, A.; Sampels, M. Hybrid Metaheuristics, v. 6373 of Lecture Notes in Computer Science, p. 160-173.

Toth, P., D. Vigo. (2002) The Vehicle Routing Problem. Monographs on Discrete Mathematics and Applications (SIAM), Philadelphia.

Wang, F.; Tao, Y.; Shi, N. (2009) A survey on vehicle routing problem with loading constraints. In: International Joint Conference on Computational Sciences and Optimization, p. 602-606.

Zachariadis, E. E.; Kiranoudis, C. T.; Tarantilis, C. D. (2009) A Guided Tabu Search for the Vehicle Routing Problem with twodimensional loading constraints. European Journal of Operational Research, v. 195, n. 3, p. 729-743.

Zachariadis, E. E.; Tarantilis, C. D.; Kiranoudis, C. T. (2013) Integrated distribution and loading planning via a compact metaheuristic algorithm. European Journal of Operational Research, v. 228, p. 56-71.
Wei, L., Zhang, Z., Zhang, D., Lim, A. (2015) A variable neighborhood search for the capacitated vehicle routing problem with two-dimensional loading constraints. European Journal of Operational Research, v. 243, n.3, p. 798-814. 